\title{
Avaliação da Atividade Antioxidante pela Inibição da Peroxidação Lipídica e Quelação de Íon Ferro de Extratos Metanólicos de Azadirachta indica (NEEM)
}

\author{
Maiara Fabiana Peruzzi ${ }^{1}$, Amanda Rafaela Luciano Breganó ${ }^{1}$, Clara Ruiz de Souza ${ }^{1}$, \\ Fernanda Arduíno ${ }^{1}$, José Carlos Duarte ${ }^{1}$, Marcela Maria Baracat ${ }^{1}$, Rubia \\ Casagrande $^{1}$ e Sandra Regina Georgetti ${ }^{1}$
}

\author{
${ }^{1}$ Universidade Estadual de Londrina (UEL) - Departamento de Ciências Farmacêuticas, \\ Caixa Postal 791 - CEP 86038-350, Londrina - Paraná - E-mail: (maiaraperuzzi@gmail.com)
}

\section{RESUMO}

Azadirachta indica, também conhecida como NEEM, é uma espécie vegetal que tem sua origem na Índia, mas com grande distribuição geográfica incluindo países tropicais. Vários estudos demonstraram que a mesma possui compostos fenólicos, os quais são responsáveis pela sua atividade antioxidante. Levando em consideração que em vários tipos de doenças, estão envolvidos danos oxidativos mediados por espécies reativas de oxigênio (EROS), este trabalho teve como objetivo avaliar e comparar as atividades inibidoras da peroxidação lipídica dependente de $\mathrm{Fe}^{+2}$ e a queladora de ín ferro dos extratos metanólicos das folhas da A. indica obtidos por agitação em shaker e por turboextração.

Palavras-chave: Antioxidante; Radicais livres; Peroxidação lipídica.

\section{INTRODUÇÃO}

Atualmente existe um grande interesse no estudo dos antioxidantes devido, principalmente, às descobertas sobre o efeito dos radicais livres no organismo. A oxidação é parte fundamental da vida aeróbica e do nosso metabolismo e, assim, os radicais livres são produzidos naturalmente ou por alguma disfunção biológica. Esses radicais livres cujo elétron desemparelhado encontra-se centrado nos átomos de oxigênio ou nitrogênio são denominados espécies reativas de oxigênio (EROs) ou espécies reativas de nitrogênio (ERNs), respectivamente (BARREIROS; DAVID; DAVID, 2006).

$O$ desequilíbrio entre as EROs e os mecanismos de defesa antioxidantes leva à instalação do estresse oxidativo. Esse processo se caracteriza pela oxidação das biomoléculas com consequente perda de suas funções biológicas e/ou desequilíbrio homeostático, cuja manifestação é o dano oxidativo potencial contra células e tecidos (BARBOSA et al., 2010).

Diante disso, o uso de produtos naturais com potencial antioxidante vem fornecendo novas perspectivas para a prevenção de danos oxidativos mediados pelos radicais livres (MEENA et al., 2012).

Análises fitoquímicas da $A$. indica demonstraram a existência de altas concentrações de compostos fenólicos que são os responsáveis pela variação da atividade antioxidante da planta (MAHMOUND; SHOLIB, 2008). 
Este trabalho teve como objetivo, avaliar a atividade inibidora da lipoperoxidação (LPO) induzida por $\mathrm{Fe}^{+2}$, e também, a capacidade queladora de íon ferro, de extratos metanólicos obtidos por agitação em shaker e por turboextração de folhas da $A$. indica, para se compreender com mais detalhes seu mecanismo antioxidante, e assim ter uma possível aplicação terapêutica de seus extratos em patologias relacionadas com danos oxidativos

\section{MATERIAL E MÉTODOS}

Obtenção dos extratos metanólicos de Azadirachta indica

As folhas de $A$. indica foram secas em estufa a $40^{\circ} \mathrm{C}$ e trituradas utilizando-se liquidificador industrial. Em seguida, os extratos metanólicos em diluição (1:10 p/v) foram obtidos por agitação em shaker por 3 dias ou por turboextração. As misturas resultantes foram filtradas à vácuo para a obtenção do extrato metanólico de NEEM obtido por agitação em shaker (ES) e por turboextração (ET) que foram utilizadas para a análise antioxidante.

\section{Determinação da atividade inibidora da peroxidação lipídica induzida por $\mathrm{Fe}^{+2}$}

A medida da atividade inibidora de peroxidação lipídica induzida por $\mathrm{Fe}^{+2}$ foi determinada pela diminuição da formação de malondialdeido (MDA), um produto secundário da peroxidação lipídica. (CASAGRANDE et al., 2006) $\mathrm{Em} 1 \mathrm{~mL}$ de meio de reação contendo: $130 \mathrm{mM}$ de $\mathrm{KCl}$ e $10 \mathrm{mM}$ de Tris- $\mathrm{HCl} \mathrm{pH} 7,4$ (meio I), foram adicionados $10 \mathrm{mM}$ de citrato de sódio, $20 \mu \mathrm{L}$ de diferentes concentrações dos extratos, suspensão de mitocôndria extraída de fígado de rato ( $1 \mathrm{mg}$ de proteína/mL de suspensão) e $50 \mu \mathrm{M}$ de sulfato ferroso amoniacal. A reação foi incubada a $37^{\circ} \mathrm{C}$ por 30 minutos. Para determinação do MDA formado, $1 \mathrm{~mL}$ de ácido tiobarbitúrico (TBA) $1 \%$ (preparado em NaOH 0,05M), 0,1mL de $\mathrm{NaOH} 10 \mathrm{M}$ e $0,5 \mathrm{~mL}$ de $\mathrm{H}_{3} \mathrm{PO}_{4} 20 \%$ foram adicionados, em seguida incubou-se a reação por 20 minutos a $80^{\circ} \mathrm{C}$. $\mathrm{O}$ complexo MDA-TBA foi extraído com $2 \mathrm{~mL}$-butanol as amostras foram centrifugadas a 3000 rpm por 10 minutos, e foi medida a absorbância do sobrenadante por espectrofotometria em $535 \mathrm{~nm}$. O ensaio foi realizado em triplicata.

\section{Determinação da atividade queladora do ín ferro}

A quelação do íon ferro pelos ES e ET foi determinada utilizando a batofenantrolina (BPS) um forte quelador de íon ferro. Para a medida da atividade queladora, $20 \mu \mathrm{L}$ de cada extrato em diferentes concentrações e $10 \mu \mathrm{L}$ de sulfato ferroso amoniacal $50 \mu \mathrm{M}$ foram adicionadas em $2 \mathrm{~mL}$ de meio I. Após 15 minutos de incubação à temperatura ambiente, adicionou-se $10 \mu \mathrm{L}$ de BPS $0,2 \mathrm{mM}$. A reação foi incubada novamente por 15 minutos à temperatura ambiente e a absorvância foi determinada pela mudança colorimétrica em 530 e $700 \mathrm{~nm}$ (UYEMURA, 2007). Todas as medidas foram realizadas em triplicata.

\section{Análise estatística dos resultados}

As concentrações dos extratos de Azadirachta indica que inibem o processo oxidativo em $50 \%$ ( $\mathrm{IC}_{50}$ ) foram estimadas pelo software GraphPad Prism ${ }^{\circledR}$, versão 4.00 de abril de 2003, utilizando uma curva hiperbólica (one site binding hyperbola). Os resultados foram expressos como média \pm erro padrão médio (EPM).

\section{RESULTADOS E DISCUSSÃO}

Entre os alvos biológicos do estresse oxidativo, os lipídios estão entre as principais biomoléculas envolvidas (UYEMURA, 2007). A inibição da LPO foi concentração dependente

Universidade Estadual de Londrina - Rodovia Celso Garcia Cid, Pr 445, Km 380 - Campus Universitário Caixa Postal 10.011 CEP 86057-970 Centro de Ciências Exatas - Departamento de Bioquímica e 
para ambos os extratos. A linearidade do ES e do ET apresentou-se entre 0,04 e 3,0 $\mathrm{LL} / \mathrm{mL}$, com valores de $\mathrm{IC}_{50}$ de $0,972 \mu \mathrm{L} / \mathrm{mL}$ e $1,035 \mu \mathrm{L} / \mathrm{mL}$, respectivamente. As porcentagens máximas de inibição da LPO foram de $91,5 \%$ para o ES e de $93,27 \%$ para o EOT utilizando a concentração de $6,0 \mu \mathrm{L} / \mathrm{mL}$ no meio reacional. Para a atividade queladora de $\mathrm{Fe}^{2+} \mathrm{a}$ linearidade encontrada foi de $0,5-5,0 \mu \mathrm{L} / \mathrm{mL}$ para ES e de $0,5-2,5 \mu \mathrm{L} / \mathrm{mL}$ para $\mathrm{ET}$. Em adição, ambos os extratos apresentaram atividade concentração-dependente com valores de $\mathrm{IC}_{50}$ de $3,17 \mu \mathrm{L} / \mathrm{mL}$ e $2,15 \mu \mathrm{L} / \mathrm{mL}$ para ES e ET, respectivamente. Estes resultados demonstram que ambos os extratos foram eficientes na diminuição dos produtos finais da lipoperoxidação e na quelação de ferro. Os resultados também demonstraram que houve diferença de atividade entre os extratos dependendo da metodologia antioxidante avaliada, indicando que o método de preparo pode influenciar no tipo e quantidade de compostos presentes no extrato gerando diferente ação antioxidante.

\section{CONCLUSÕES}

Os resultados sugerem que a atividade antilipoperoxidativa apresentada pelos extratos metanólicos de NEEM pode ocorrer por sequestro de radicais livres que são formados durante a peroxidação, como também, pela capacidade dos extratos em reduzir a disponibilidade do íon ferro, prevenindo a formação dos radicais lipídicos e a propagação da peroxidação lipídica. Em adição, verificou-se que o método de obtenção do extrato pode influenciar na atividade antioxidante da planta. Assim sendo, o mesmo apresentou uma possível aplicação em patologias relacionadas com a peroxidação lipídica.

Agências de Fomento: Fundação Araucária e CNPq.

\section{REFERÊNCIAS}

BARBOSA, K.B.F; COSTA, N.M.B; ALFENAS, R.C.G; DE PAULA, S.O; MINIM, V.P.R; BRESSAN, J. Estresse oxidativo: conceito, implicações e fatores modulatórios. Revista de Nutrição, 23, p.629-643, 2010.

BARREIROS, A.L.B.S; DAVID, J.M; DAVID, J.P. Estresse oxidativo: relação entre geração de espécies reativas e defesa do organismo. Química Nova, 29, p.113-123, 2006.

CASAGRANDE, R.; GEORGETTI, S.R.; VERRI, W.A., JR; JABOR, J.R.; SANTOS, A.C.; FONSECA, M.J.V. Evaluation of functional stability of quercetin as a raw material and in different topical formulations by its antilipoperoxidative activity. AAPS PharmSciTech, 7,p.E1-E8, 2006.

MAHMOUD, M.F.; SHOEIB, M.A. Sterilant and oviposition deterrent activity of neem formulations on Peach fruit fly Bactrocera Zonata (Saunders) (Diptera: Tephritidae). Journal of Biopesticides, 1, p.177-181, 2008.

MEENA, H.; PANDEY, K.H.; PANDEY, P.; ARYA, M.C.; AHMED, Z. Evaluation of antioxidant activity of two important memory enhancing medicinal plants Baccopa monnieri and Centella asiatica. Indian Journal of Pharmacology, 44, p.114-117, 2012.

UYEMURA, V. T. Efeito do extrato de Tamarindus indica L. sobre a transição da permeabilidade de membrana em mitocôndrias isoladas de fígado de rato e atividade antioxidante in vitro. Tese de Doutorado, Universidade de São Paulo, Ribeirão Preto, Brasil, 2007.

Universidade Estadual de Londrina - Rodovia Celso Garcia Cid, Pr 445, Km 380 - Campus Universitário Caixa Postal 10.011 CEP 86057-970 Centro de Ciências Exatas - Departamento de Bioquímica e Biotecnologia Fone +55 (43) 3371.4270 - biq@uel.br 